\title{
Consequencies of the new intensity formula in many optical spectroscopy fields
}

\author{
Bo Thelin \\ Solarphotonics HB Granitvägen 12B 75243 Uppsala Sweden
}

\begin{abstract}
This paper is an extended review paper about the use of a new intensity formula in optical emission spectroscopy, atmospheric physics and astronomy. The laboratory data of atomic-and ionic spectra from different light sources do support the new formula. In the atmospheric field dominant light mechanisms in aurora have been revealed, which follow the new formula. The inverse of this formula can also be used to show the photoelectric effect for many elements.

In astronomy new methods have been developed of determining electron-and effective temperature, density and mass of stars with the new formula as a basis, which are in accordance with literature values. An investigation of the Balmer lines in the sun shows that they do follow the new formula. Therefore the new formula is important in the sun and the stars.

Keywords: Optical emission spectroscopy, Analytical Chemistry, Light sources, Spectral tables UV-IR, Atmospheric emissions, Auroral spectroscopy, Astrophysics, Linear relationships, Photoelectric effect, Star temperature determinations, Star density determinations and Star mass determinations.
\end{abstract}

\section{Introduction}

The work to be presented in this paper is an extended review paper from many papers in the literature concerning a new intensity formula in optical emission spectroscopy. It originates from a certain discovery made by the author and Dr.Sten Yngström during the 1980s, while working with auroral spectroscopy at the Swedish Institute of Space Physics in Kiruna. The data of interest were achieved by using a new computerized, extremely versatile photon counting spectrometer system (IDES), which was suited for the study of intensities of spectral lines.

According to a new theory by S.Yngström Ref 1 , which was created during the 1980s, the intensity I is given

$$
I=C \lambda^{-2}(\exp (-J / k T)) /(\exp (\mathbf{h} \mathbf{v} / \mathbf{k} T)-1)
$$

$\mathrm{J}$ is here the ionization energy, and $\mathrm{C}$ is a factor given by transition probabilities, number densities and sample properties. $\lambda$ and $v$ are here the wavelength and frequency of the atomic spectral line. This means that the new intensity formula consists of 4 parts : the C-factor, $\lambda^{-2}$-part, the J-dependence $\exp (-\mathrm{J} / \mathrm{k} \mathrm{T})$ and the Planck factor $1 /((\exp (\mathrm{h} v / \mathrm{kT})-1)$.

Myself and my collegue Dr.Sten Yngström have earlier presented a big number of papers in the past about this formula. We have developed three methods of analyses : the fluctuation method, the absolute intensity method and the detection limit method, which all support the new intensity formula in equation 1. Two summary papers Refs 2 and 3 about this project have earlier been published in open access journals together with many other papers in these fields. This means that they contribute to a strong evidence from the literature about this new intensity formula. It is common in the literature to find papers and data from investigations in the past supporting the new intensity formula.

In this very paper the same kind of studies about the absolute intensities will be presented for ionic spectra, which earlier were performed on atomic spectra. A new intensity formula for ions has also been presented. Ionic studies were also performed for $\mathrm{C}, \mathrm{O}$ and $\mathrm{Ne}$-ions, where a new recursion formula was presented in Ref 4.

The internal electron temperature was also determined in relation to the ionization energies. Intensity studies were also done in the ICP- plasma, where the internal electron temperature was studied at different heights in the plasma supporting the new intensity formula.

Studies from the LED-and fluorescens experiments at room temperature were also discussed in relation to the Planck profile of the new formula.

Breakdown- and X-ray experiments have also been discussed in relation to the new formula.and new formulas for these experiments were also presented.

The photoelectric effect has also been studied, giving a new formula for this effect by using the inverse of the new intensity formula. This was studied for many different elements and the alkali metals.

In the nature the new formula was studied with the fluctuation method on Ba-ions released from explosions in the upper atmosphere by using rockets. Spectrophotometers on the ground were detected the light from these 
explosions. In these investigations airglow and auroral emissions were also measured on ground measurements with spectrophotometer, where the optical emission mechanism was studied.

The stellar measurements here were based on the linear relationship found between spectrophotometer data of stars, used earlier with the absolute intensity method on at atomic-and ionic laboratory data. From these measurements it was possible to determine electron -and effective temperature, density and mass of stars in accordance with literature data. The Balmer spectral lines were also studied with the new formula.

\section{2a The fluctuation method}

\section{Laboratory experiments supporting the new formula}

The first method which was developed supporting the intensity formula with an exponential $(\mathrm{J}+\mathrm{h} v)$ term is the fluctuation method. This method concerns the study of spectral line intensity ratio fluctuations in Refs ( 5 - 10 ), where many different optical light sources were used. By forming the ratio between the intensities of two simultaneously measured lines from the same sample and by using logarithmic differentiation, we obtain the following expression

$\mathbf{d}\left(\mathbf{I}_{\mathrm{mn}}^{\mathrm{a}} / \mathbf{I}_{\mathrm{kl}}^{\mathrm{b}}\right) /\left(\mathbf{I}_{\mathrm{mn}}^{\mathrm{a}} / \mathbf{I}_{\mathrm{kl}}^{\mathrm{b}}\right)=\mathbf{d}\left(\mathbf{C}_{\mathrm{mn}}^{\mathrm{a}} / \mathbf{C}_{\mathrm{kl}}^{\mathrm{b}}\right) /\left(\mathbf{C}_{\mathrm{mn}}^{\mathrm{a}} / \mathbf{C}_{\mathrm{kl}}^{\mathrm{b}}\right)+(\mathbf{1} / \mathbf{k} \mathbf{T})(\mathbf{d} \mathbf{T} / \mathbf{T}) \mathbf{D}(\mathbf{E})$

where $\mathrm{D}(\mathrm{E})=\mathrm{J}^{\mathrm{a}}-\mathrm{J}^{\mathrm{b}}+\mathrm{h} v_{\mathrm{mn}}^{\mathrm{a}}-\mathrm{h} v_{\mathrm{kl}}^{\mathrm{b}}$ and $\mathrm{d}\left(\mathrm{I}_{\mathrm{mn}}^{\mathrm{a}} / \mathrm{I}^{\mathrm{b}}{ }_{\mathrm{kl}}\right) /\left(\mathrm{I}_{\mathrm{mn}}^{\mathrm{a}} / \mathrm{I}_{\mathrm{kl}}^{\mathrm{b}}\right)$ is named $\mathrm{R}$ value. Mathematically this formula is a straight line, which can be seen in Fig ( 1 ) from a hollow cathode experiment. In this figure fluctuation data $R$ versus $D(E)=J^{a}-J^{b}+h v_{m n}^{a}-h v_{k l}^{b}$. (difference of ionization energy plus photon energy) were used from fifteen steel samples in a hollow cathode lamp. Seventeen elements were studied in this graph giving a correlation coefficient of 0.90 .

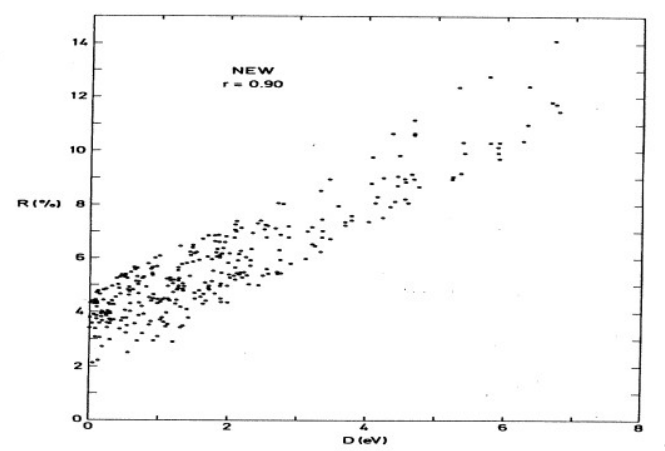

FIG 1 Plot of fluctuation data R versus D (E) $=J^{\mathrm{a}}-\mathrm{J}^{\mathrm{D}(\mathrm{b})}+\mathrm{h} v_{\mathrm{mn}}^{\mathrm{a}}-\mathrm{h} v_{\mathrm{kl}}^{\mathrm{b}}$. (difference of ionization energy plus photon energy) from fifteen steel samples used in a hollow cathode lamp. Seventeen elements were studied in this graph. (Reproduction from Ref 10)

\section{2b The absolute intensity method}

\section{Atomic spectra}

A summary of this second method is presented here and concerns about studying absolute intensity of spectral lines. This method has been presented in several papers earlier Refs 10, 11 and 12). In this very paper this method uses spectral tables from NBS as a basis. According to this method it has shown to be possible to obtain linear relationships by studying the logarithmic expression :
$\ln \left(\mathbf{I} \lambda^{2}\right)=-(h v / k T)(1+(k T / h v) \ln (1-\exp (-h v / k T)))$

which was developed from equation 1. This investigation was based on NBS intensity tables on arc measurementsRef 13 and 14 . The points in this graph represent the mean values of many spectral lines in a small wavelength interval. A graph like this is shown in Fig 2 , which is a graph of C(I)(atoms) and shows linearity over $14 \mathrm{eV}$, which is a nice result with a correlation coefficient $\mathrm{r}=-0.97$ and shows that the carbon atoms do to follow this formula.

In this method $\ln \left(\mathrm{I} \lambda^{2}\right)$ was plotted versus $\mathrm{h} v(1+\theta / \mathrm{h} v \ln (1-\exp (-\mathrm{h} v / \theta))) \mathrm{eV} \quad$ for 17 atomic elements. Each intensity value here is the mean value of many individual values. By forming the maximum between the difference between $\ln I \lambda^{2}$ and $\ln \lambda^{2}$ the following formula will be the basic equation in this method of analysis.

$$
\ln \left(I_{\max } \lambda^{2} \max \right)=\text { const. }-1.6 \mathrm{~J} / \mathbf{h} v_{\max }
$$

This graph is seen in Fig 3 , where $\ln \left(\mathrm{I}_{\max } \lambda^{2}{ }_{\text {max }}\right)$ has been plotted versus $1.6 \mathrm{~J} / \mathrm{h} v_{\max }$

$=\mathrm{J} / \theta$ for 17 elements, where $\theta=\mathrm{k} \mathrm{T}_{\mathrm{e}}$ (electron temperature). $\mathrm{J}$ denotes table value of ionization energy. This graph forms a good linear relationship, where $h v_{\max }=1.6 \theta$. This means that this graph is a strong support of the new intensity formula, based on the new theory. It is also possible to measure the internal electron temperature (around $2 \mathrm{eV}$ ) for different elements, which is shown in Table 1. These values of the electron temperature are supported by secondary electron temperature values from the literature in Ref 15 . 


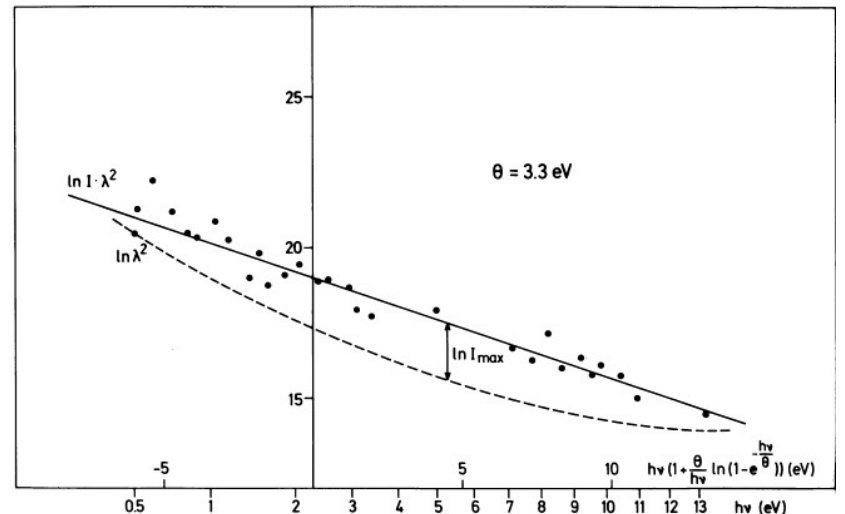

FIG $2 \ln \left(\mathrm{I} \lambda^{2}\right)$ plotted against $\mathrm{h} v(1+(\mathrm{k} \mathrm{T} / \mathrm{h} v) \ln (1-\exp (-\mathrm{h} v / \mathrm{k} T))$ e $\mathrm{V}$ for carbon C ( I ) data. Intensity data from intensity tables were used. (Reproduction from Ref 11 )

$\begin{array}{lcc}\text { Element } & \begin{array}{c}\text { Table 1 } \\ \mathbf{J}(\mathbf{e V})\end{array} \\ \mathrm{Cs} & 3.89 & \\ \mathrm{Na} & 5.14 & 1.6 \\ \mathrm{Ba} & 5.20 & 1.9 \\ \mathrm{Li} & 5.39 & 1.8 \\ \mathrm{Ca} & 6.11 & 2.1 \\ \mathrm{Yb} & 6.25 & 2.1 \\ \mathrm{Sc} & 6.70 & 2.1 \\ \mathrm{Cr} & 6.76 & 2.3 \\ \mathrm{Ti} & 6.83 & 2.1 \\ \mathrm{Sn} & 7.33 & 2.1 \\ \mathrm{Mo} & 7.38 & 2.3 \\ \mathrm{Mn} & 7.43 & 2.3 \\ \mathrm{Ag} & 7.57 & 2.1 \\ \mathrm{Ni} & 7.63 & 2.1 \\ \mathrm{Fe} & 7.86 & 2.1 \\ \mathrm{Co} & 7.88 & 2.2 \\ \mathrm{Pt} & 9.0 & 2.1\end{array}$

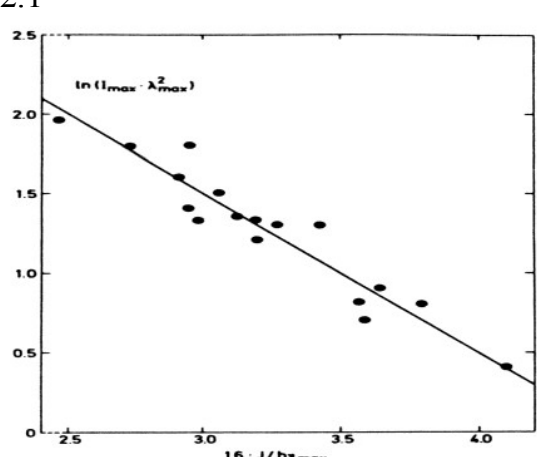

Fig $3 \ln \left(\mathrm{I}_{\max } \lambda_{\max }^{2}\right)$ plotted versus $(1.6 \mathrm{~J}) / \mathrm{h} v_{\max }$ for seventeen elements from the NBS tables in ( 13 ) and (14) (Reproduction from Ref 10 )

\section{2c The method of ionic spectra}

The intensity formula for ions has a similar appearance as equation 1 and is shown in equation 5 . ( Refs 4, 17 and 18 ) This formula include ionization energies for the first and second ionization energy, which has been proposed earlier in the detection limit method Ref $16 . \mathrm{C}$ is a factor given by transition probabilities, number densities and sample properties. $\lambda$ and $v$ are here the wavelength and frequency of the ionic spectral line. The ionic intensity formula has the following appearance :

$$
I=C \lambda^{-2}\left(\exp \left(-\left(J_{1}+J_{2}\right) / k T\right)\right) /(\exp (h v / k T)-1)
$$

To show the validity of equation 5 with the short version of this method $\ln \left(\mathrm{I} \lambda^{2}\right)$ was plotted versus $\mathrm{h} v($ $1+\theta / h v \ln (1-\exp (-\mathrm{h} v / \theta))) \mathrm{eV}$ for CII and is shown in Fig 4 and is similar to the graph of CI in Fig 2. Similar graphs were also obtained for OI,OII, NeI and NeII, which gave linear graphs $(\mathrm{r}=-0.98$ for OI and $\mathrm{r}=-0.95$ for 
OII and $\mathrm{r}=-0.98$ for NeI and $\mathrm{r}=-0.99$ for NeII). From these graphs $\ln \left(\mathrm{I}_{\max } \lambda^{2}{ }_{\max }\right)$ was plotted versus $1.6\left(\mathrm{~J}_{1}+\mathrm{J}_{2}\right)$ $/ \mathrm{h} v_{\max }=\left(\mathrm{J}_{1}+\mathrm{J}_{2}\right) / \theta$ in the same way as for atoms which is seen above. The points will follow an expression in equation 6 for ions which is similar to equation 4 for atoms.

$\ln \left(I_{\max } \lambda_{\max }^{2}\right)=$ const. - $1.6\left(\mathbf{J}_{1}+\mathbf{J}_{2}\right) / \mathbf{h} v_{\max }$

Equation 5 includes ionization energies for the first and second ionization energy.

These values fit well with secondary electron temperature values from the literature Ref 15 .

A similar plot to Fig 3 for atoms has also been done for ions of 11 elements, which is seen in Fig 5. The electron temperatures are about $4 \mathrm{eV}$ for ions which fit well with energy distributions of secondary electrons from different experiments in the literature

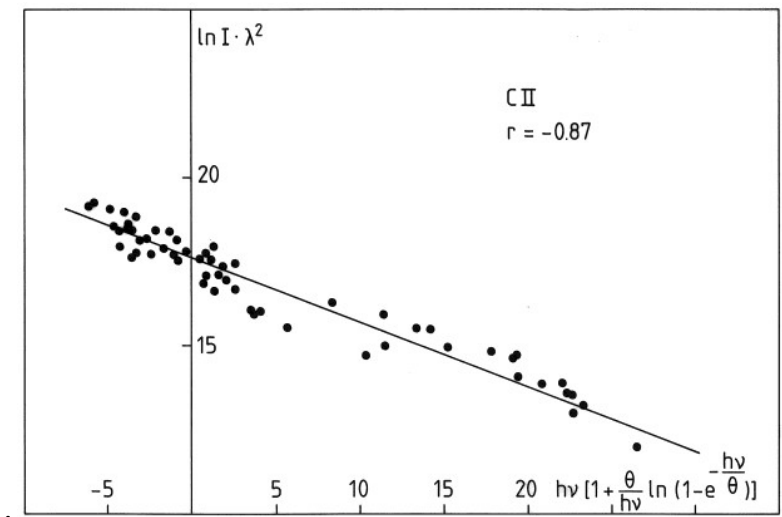

FIG $4 \ln \left(\mathrm{I} \lambda^{2}\right)$ plotted against $\mathrm{h} v(1+(\mathrm{kT} / \mathrm{h} v) \ln (1-\exp (-\mathrm{h} v / \mathrm{kT})) \mathrm{eV}$ for carbon $\mathrm{C}(\mathrm{I}$ I ) ions. Intensity data from intensity tables were used. ( Reproduction from Ref 4 )

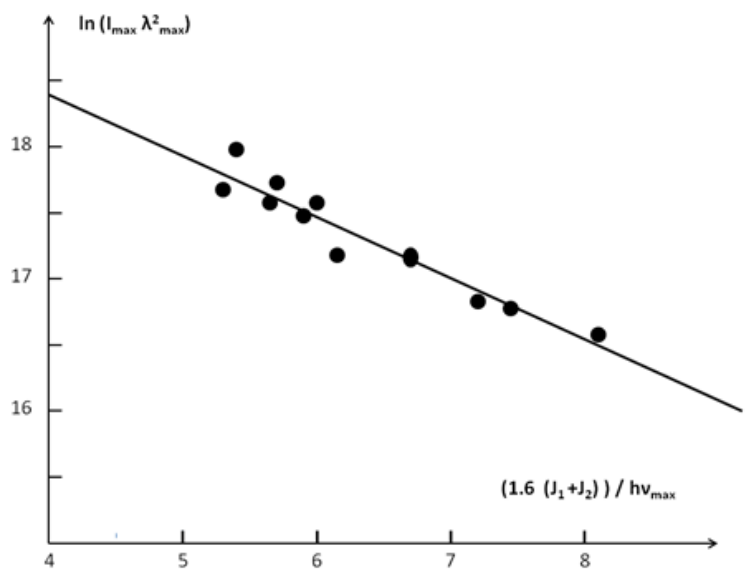

Fig $5 \ln \left(\mathrm{I}_{\max } \lambda^{2}{ }_{\max }\right)$ plotted versus $\left(1.6\left(\mathrm{~J}_{1}+\mathrm{J}_{2}\right)\right) / \mathrm{h} v_{\max }$ for eleven ionic elements from the NBS tables. Reproduction from Ref 4 )

\section{2d. This method of spectra of ions with higher ionization energies}

The intensity formula for the ions with higher ionization energies follow similar linear relationships as Figs 2 and 4. These can be seen for oxygen in Fig 6 for, OV and OVI with very good correlations in Ref 4. The variance of the slopes of the lines depend of the difference of the electron temperatures between the graphs. The higher ionization energy the lower slope.

The new intensity formula for ionic line spectra can be approximately written for ions of the $(\mathrm{r}+1)$ : th stage :

$I_{r+1} \propto N_{r+1} \exp \left(-\left(J_{r+1}+h v\right) / k T\right)=N_{r} \exp \left(-J_{r} / k T\right) \exp \left(-\left(J_{r+1}+h v\right) / k T\right)$

In this way it seems to be possible to build up an approximate recursion formula of the $\mathrm{k}:$ th

stage : $\quad \mathbf{I}_{\mathbf{k}} \propto \mathbf{N}_{1} \exp \left(-\left(\sum\left(\mathbf{J}_{\mathrm{r}}\right)_{\mathrm{r}=1}^{\mathrm{r}=\mathrm{k}}+\mathbf{h v}\right) / \mathbf{k T}\right)$

Therefore a complete recursion formula for the ionic spectra could be written similar to equation 1 for atomic spectra. 


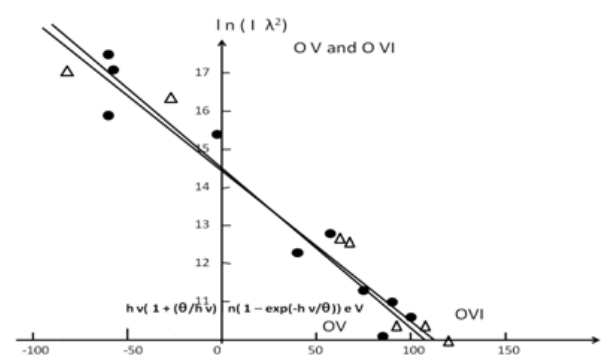

Fig $6 \ln \left(\mathrm{I} \lambda^{2}\right)$ plotted against $\mathrm{h} v(1+(\theta / \mathrm{h} v) \ln (1-\exp (-\mathrm{h} v / \theta))$ e $\mathrm{V}$ for oxygen $\mathrm{O} \mathrm{V}$ and OVI data (ions). Intensity data from intensity tables were used. ( Reproduction from Ref 4 )

\section{2e The detection limit method}

A third method of analysis has been developed concerning the detection limits of ionic spectral lines in an inductively coupled plasma experiment Ref 16. A table of transition probabilities of spectral lines in an arc experiment was used in this work. The results from that analysis of detection limit data are very consistent with the new spectral line expression of equation 1. In this paper a method was developed to calculate transition probability rates from established tables according to the new theory of spectral line intensities.

\section{2f. Inductively coupled plasma ICP}

In a paper by Blades et al in Ref 19 an ICP- temperature investigation was carried out for different elements on different heights $(\mathrm{H})$ of the ICP-plasma. A straight line was obtained, where the heights of different spectral lines(peak values) from different elements were plotted versus the normal temperature. According to this graph the $\mathrm{I}(\max )$ from these lines are inversely proportional to the heights $(\mathrm{H})$ of the lines. From that graph(Fig 19, p.861) in this reference) it is possible to plot the height versus $(\mathrm{J}+\mathrm{h} v)$, which is linear and shows a direct proportionality between height and $(\mathrm{J}+\mathrm{h} v)$ and is shown in Fig 7. Because I(max) is inversely proportional to $\mathrm{H}$, means that $\mathrm{I}(\mathrm{max})$ is inversely proportional to $(\mathrm{J}+\mathrm{h} v)$ which is in accordance to the equation 1 . Refs 2 and 3.

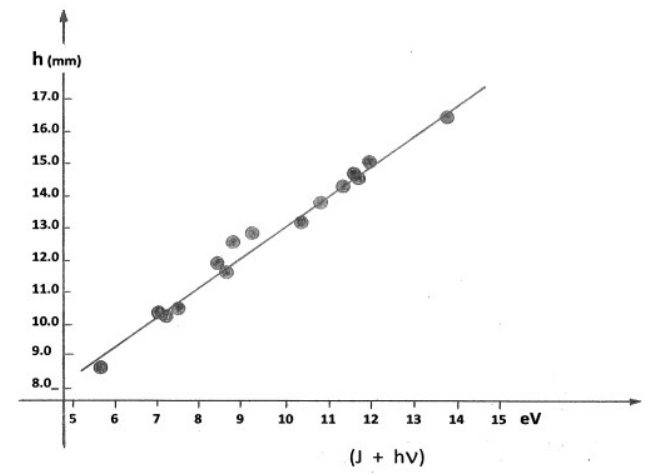

FIG 7 This graph shows a linear relationship between height $(\mathrm{H})$ in the ICP - plasma and $\mathrm{J}+\mathrm{h} v$ for different elements studied. ( Reproduction from Ref 2 )

\section{2g. LED lamps, Photoluminiscence and Electroluminiscence}

Planck factor in the formula is also strongly supported by the intensity profile $I=f(\lambda)$ of the LEDlamps .These lamps are working at low temperature as in Ref 20 (Fig 3 and 4, p.5087) and Ref 21 (Fig $6 b, p .8267)$. These curves were coming from experiments in room temperature and far below(26-200K) from photoluminisscence FL-experiments. These two references constitute good examples of the fact that emissions coming from low temperature light sources show Planck factor profiles coming from discrete emissions. The reason for this is the fact that the temperature is here too low to emit continuous radiation. These observations agree well with the ideas of this paper, where the Planck profile from the discrete emission lines is clearly seen here.

In many electroluminescence papers like Ref 22 the envelope of these emission peaks seems to follow a Planck factor profile according to equation 1 . The deep minima between the emission peaks, show that the emissions are mostly coming from discrete emissions and not from continuous emissions. Therefore the Planck factor profile here seems to originate from equation 1 for discrete emissions. 


\section{2g. Breakdown experiment with laser}

In Fig 9.9 ,p.737 of Ref 23 the breakdown pressure is plotted versus ionization energy for some atomic and molecular gases. In this investigation the laser power was fixed. The $\mathrm{C}$-factor includes the number densities of a gas, which means the pressure in this experiment. In this experiment the pressure is proportional to the ionization energy. This fact is in accordance to equation 1 because the $\mathrm{C}$-factor can be expressed as approximately

as a breakdown formula Ref 3 .

$$
\mathbf{C}=\mathbf{I} \lambda^{2} \exp ((\mathbf{J}+\mathbf{h} v) / \mathbf{k} \mathbf{T})
$$

\section{2h. X-ray experiments}

This dependence has been studied earlier Ref 24 (Fig 2-9,p.93) and Ref 25 (Fig 12, p.349) in the X-ray field, where

$$
\text { I }(\lambda)=\text { const } \lambda^{-2}
$$

for the X-ray emissions. In this wavelength region the $h v$-value is very big which makes the Planck factor around 1 . If $\mathrm{kT} \approx \mathrm{h} v$, then equation 10 is achieved from equation 1.For a specific element this expression is in accordance with equation 1 as an X-ray formula Ref 3 .

\section{2i The photoelectric effect}

In this section new results about the photoelectric effect have been obtained by using the new intensity formula in optical emission spectroscopy. By using the inverted form of this formula good correlation between the current and frequency has been obtained from photoelectric experiments at different field strengths. At photoelectric and thermionic experiments from different elements the importance of the ionization energy in the photoelectric effect has been demonstrated.

As this intensity formula deals with number of emitting photons from a plasma, it has also shown to be possible to use it on the reverse process, where incoming photons emit electrons into a current. An experimental proof of that have been shown earlier by the author in Ref 26. A resume of this paper is shown here.

A fact which is important in this very paper about the photoelectric effect is a relationship between current and frequency. The usual appearance from the literature of this experimental relationship is an $i=e^{v}$-structure, where the current ( $y$-axis) is an exponential function of the frequency $(x$-axis), and starts from the $v_{0}$ (threshold) a bit in on the $v$-axis. This experimental fact is what the different theories have tried to fit, with some difficulties according to their own opinions.

By using the approximate form of equation 1 , we can express the $\mathrm{C}$-factor as approximately

$$
\mathrm{C}=\mathrm{I} \boldsymbol{\lambda}^{2} \exp \left((\mathrm{J}+\mathbf{h v}) / \mathbf{k T} \mathbf{e}_{\mathrm{e}}\right)
$$

where a very good fit could be obtained as a new photoelectric formula. The $\mathrm{C}$-factor is here proportional to the current in the photoelectrical effect. Such a nice fit can be seen in Fig 8, from a photoelectrical experiment with potassium at different field strengths. In this fit, current ratios have been calculated according to equation 11 where the current $\mathrm{i} \alpha \mathrm{C}$.In this way only the $v$-dependence of the $\mathrm{C}$-factor(current) appeared more clearly. This data were coming from Fig 3-46 p.111 of Ref 27 and is demonstrating the influence of electric field on the photoelectric threshold of thin layers of potassium on tungsten.

This data current ratios were then calculated from the data and compared with the current from equation 11. This can be seen in equation 12 .

$i_{k} / i_{k-1}=\left(\exp \left(h v_{k} / k T_{e}\right)-1\right)\left(h v_{k-1}\right)^{2} /\left(\exp \left(h v_{k-1} / k T_{e}\right)-1\right)\left(h v_{k}\right)^{2}$
$\approx\left(v_{k-1} / v_{k}\right)^{2} \exp \left(h / k T_{e}\left(v_{k}-v_{k-1}\right)\right)$

Nice correlation between experimental and theoretical values were obtained at two different field strengths ( A and $\mathrm{B})$ in these experiments.

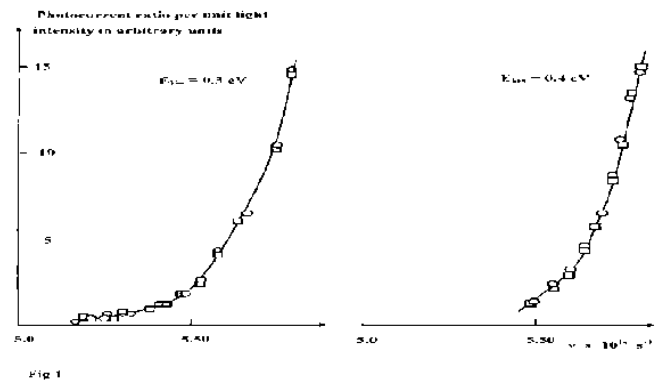

Fig 8 The $v$ - dependence of the photocurrent ratios between literature experiment(circles) and the new theory (squares)(inversed intensity formula) Results at different electron energies are shown. (Reproduction from Ref 26 ) 
The influence of the J-term of equation 11 on the photoelectric effect, has also been seen in several ways. In Table 3-2 p.75-76 of Ref 27 photoelectric and thermionic work functions of different elements have been studied. This table has been used in Fig 9, where the ionization energies $(\mathrm{J})$ have been plotted versus the threshold wavelengths of that table. Here is it possible to study the photoelectric effect of different elements. Fig 9 is similar to Fig 8 if $\mathrm{i} \alpha \mathrm{C} \alpha \mathrm{J}$ according to equation 11 but include information from 22 different elements. Fig 9 is a clear evidence of J-dependence of the photoelectric effect, where the slope of the curve increases with higher $(\mathrm{J}+\mathrm{h} v)$ - values according to equations 13 and 11 .

According to equation $13 \mathrm{~J} \alpha \mathrm{i}$ (current) which means that Fig 9 shows the usual $\mathrm{e}^{v}$-dependence between the current and frequency as in Fig 8.

If we express $J$ as a function of $\lambda$ from equation 11 we obtain :

$$
J=f\left(\lambda_{0}\right)=k T_{e} \ln \left(C / I \lambda^{2}\right)-h c / \lambda
$$

Another way to show that equation 11 seems to be important here, is the use Table 12-1,p.423 and Fig 12-8, p.424 in Ref 27 for the alkali metals. These results are shown in Fig 10 and seem to be similar to Fig 9 with the same kind of structure. In these experiments with the alkali metals the sample was well prepared and the equipment was well outgased to obtain good results.

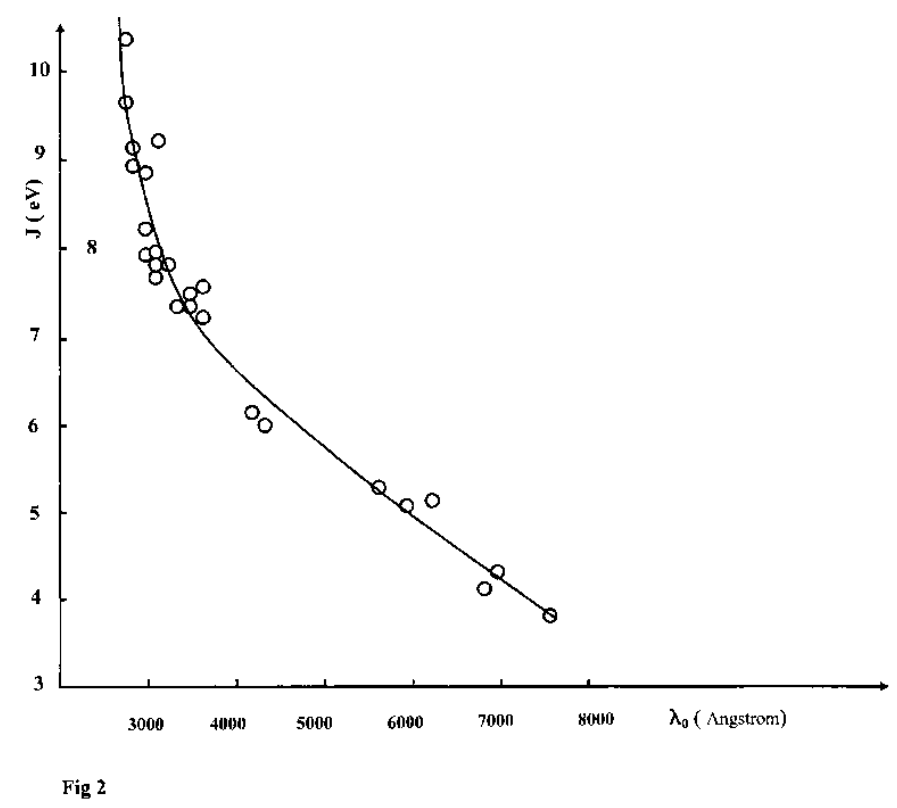

Fig 9 The influence of the ionization energy J versus the threshold wavelengths of the photoelectric effect. 22 different elements(metals) have been studied. ( Reproduction from Ref 26 )

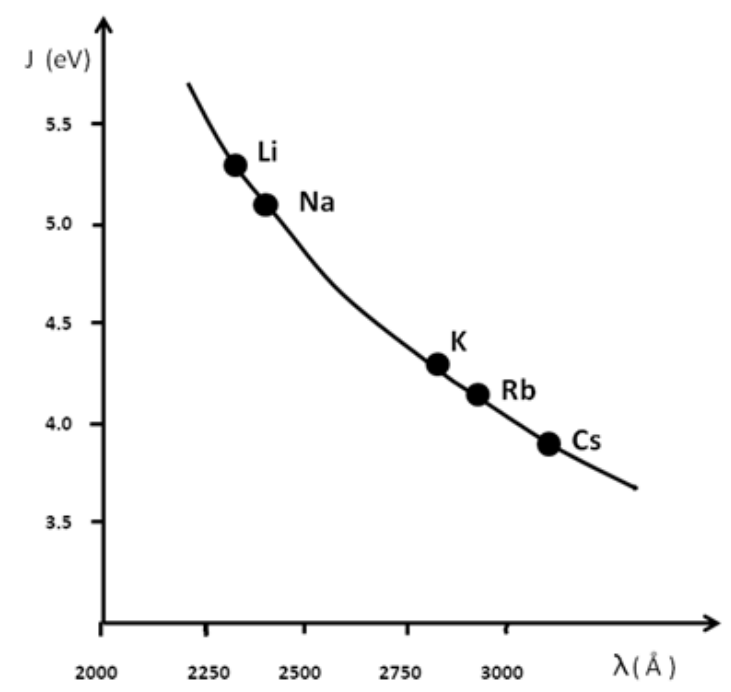

Fig 10 The influence of the ionization energy J versus the threshold wavelengths of the photoelectric effect. The alkali metals have been studied. ( Reproduction from Ref 26 ) 


\section{Atmospheric- and ionosperic emissions}

In this paper observational results about wave number dependent spectral line ratio fluctuations in the atmospheric emissions are reported. Linear relationships were obtained when spectral line ratio fluctuations (Rvalues) were plotted vs. the differencies in photon energies $\mathrm{D}(\mathrm{E})$-values when studying Ba-release Fig 11, nightglow and auroral emissions. The data used was coming from ground based spectrophotometer measurements. In the Ba-release experiment reported here a $0.5 \mathrm{~m}$ Ebert spectrophotometer was registrating two Ba-release explosions from ground at a site at the John Hopkin University. The Ba explosives were sent up by a Javelin sounding rocket from Wallops Islands Ref 29. The emission spectra of the Ba release from the explosions were recorded in the wavelength region $3490-6490 \AA$. from the ground. The data from these measurements has been used by the author with fluctuation analysis(RD-analysis) Ref 28.

In the auroral investigation a spectrophotometer SPI was used, with photoelectric registration and a photomultiplier tube. With this spectrophotometer system it is possible to choose especially interesting spectral ranges. The spectral range studies was $4200-6400 \AA$ Ref 31 . The author has done similar measurements of auroral emissions at the same wavelength range at the Institute of Space Physics in Lycksele ( Ref 30 ).

By doing such studies of auroral light it is possible to see the importance of the inelastic scattering cross section between electrons - atoms and electrons - molecules. In this way it has found to be possible to determine the mean energy of the interacting thermal electrons which are active in the different auroral phases.

This paper demonstrates that the fluctuation method( RD-graphs) from the new intensity formula seems to reveal interesting auroral results about de-excitation mechanisms. However, this electron loss process due to the excitation of rotational and vibrational states of molecules and electronic states of atoms, is an important cooling process in the ionosphere. This work also show that the new intensity formula seem to be valid in the atmospheric field too.

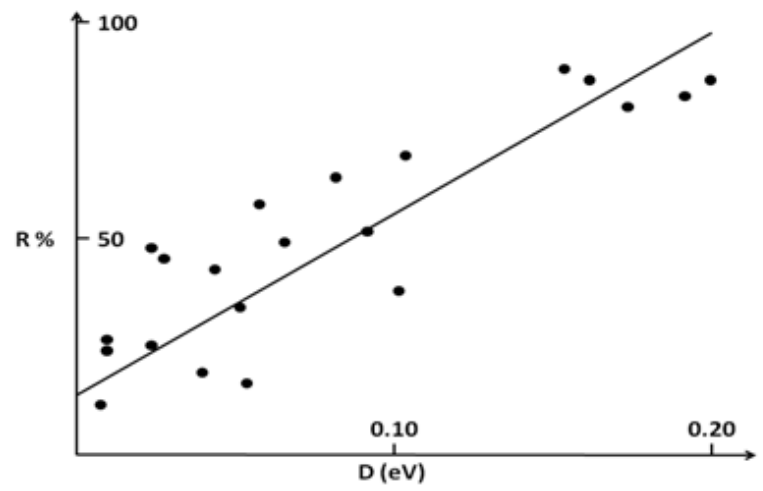

Fig 11 Fluctuation graph (RD-graph) from the Ba-release experiment ( 5 - $50 \mathrm{~s}$ after the explosion).

(Correlation $\mathrm{r}=0.84$ ) (Reproduction from Ref 28)

\section{Stellar spectral emissions}

This section is a summary of stellar measurements and analysis done by the author on data from Ref 32. These stellar optical spectra extend over the spectral classes $\mathrm{O}-\mathrm{M}$ and the photometrically well-calibrated luminosity measurements from star to star. Good temperature and luminosity coverage have been achieved. The data were digitalized from the main sequence classed $\mathrm{O} 5-\mathrm{F} 0$ and $\mathrm{F} 6-\mathrm{K} 5$ displayed in term of relative flux as a function of wavelength. The parameters that have been measured in this investigation are maximum luminosity $\mathrm{L}_{\max }$ (Rel.fluxmax) of the Planck curve. In this maximum the wavelength $\lambda_{\max }$ and the maximum frequency $v_{\max }$ were also measured.

The $\ln \left(\mathrm{L}_{\max } \lambda_{\max }{ }^{2}\right)$ values were then plotted versus $\left(1.6 \mathrm{~J}_{\text {meanvalue }} / \mathrm{h} v_{\max }\right)$ where $\mathrm{J}_{\text {meanvalue }}$ is the mean value of the ionization energies of the elements of the stars measured. To obtain a similar linear relationship for the stellar data as in Fig 3 from the spectroscopical method from Refs 10 and 11, the following luminosity data from Ref 32 and data from Table 2 were used and plotted according to equation 14

$$
\ln \left(L_{\max } \lambda_{\max }{ }^{2}\right)=\text { const }-\left(\begin{array}{ll}
1.6 & J_{\text {meanvalue }} / h v_{\text {max }}
\end{array}\right)
$$

which is similar to equation 4 for atoms.

To receive the data of Table 2 it is necessary to use a two step procedure. In the first step it is necessary to define the graph by calculating the $\mathrm{J}_{\text {meanvalue }}$ of the $\mathrm{G} 2$-star. The $\mathrm{J}_{\text {meanvalue }}$ can be

expressed in the following way:

$$
\mathbf{J}_{\text {meanvalue }}=\sum \mathbf{c}_{\mathbf{n}} \mathbf{J}_{\mathbf{n}}
$$

The $c_{n}$ is the normalized content of an element of a star. We consider the content values of G2-stars to be similar to the content values of the sun. Therefore the $c_{n}$-values of the sun have been used here. $J_{n}$ is here the ionization energy of an element of a star. This $J_{\text {meanvalue }}$ has been calculated for the sun (G2 star), which gave $J_{\text {meanvalue }}=$ $16.2 \mathrm{eV}$ according to the linear graph in Fig 12. This value is $16.2 \mathrm{eV}$, for the sun when using equation 14 
together with established chemical composition values of the sun. This means that we now have one point determined in Fig 12. A more detailed description of this analysis method of creating Fig 12 and Table 2 is described in $\operatorname{Refs}(33,34,35$ and 18 ).

The data in Fig 12 constitute a straight line in the classes $\mathrm{O} 5$ - F0 and F6 - K5 and is in accordance with the usual HR-diagram.

In equations 4 and $14 \mathrm{~h} v_{\max }=1.6 \theta$, where $\theta=$ internal electron temperature in $\mathrm{eV}$. This means that the classes O5 - F0 have higher temperature than the classes F6 - K5, which is also in accordance with the usual HRdiagram. For example a G2 star (the sun) has $\theta=1.56 \mathrm{eV}\left(\mathrm{T}_{\mathrm{e}}=18110 \mathrm{~K}\right)$.

Table 2

Determination of the electron temperature of the stars from different spectral classes

\begin{tabular}{|c|c|c|}
\hline Spectral class & $\theta(\mathrm{eV})$ & $J_{\text {meanvalue }}(\mathrm{eV}$ \\
\hline $\mathrm{K} 5 \mathrm{~V}$ & 1.44 & 15.5 \\
\hline $\mathrm{K} 4 \mathrm{~V}$ & 1.47 & 15.6 \\
\hline G9-K0 & 1.50 & 15.8 \\
\hline G6-G8 & 1.53 & 16.0 \\
\hline G1-G2 & 1.56 & 16.2 \\
\hline F8-F9V & 1.63 & 16.7 \\
\hline F6-F7V & 1.63 & 16.5 \\
\hline A9-F0V & 1.72 & 16.9 \\
\hline A8 & 1.75 & 17.1 \\
\hline A5-A7 & 1.81 & 17.5 \\
\hline A1-A3 & 1.84 & 17.6 \\
\hline B6V & 1.88 & 17.8 \\
\hline B3-B4V & 1.94 & 18.0 \\
\hline O7-B0V & 1.97 & 18.1 \\
\hline $\mathrm{O} 5 \mathrm{~V}$ & 2.00 & 18.2 \\
\hline
\end{tabular}

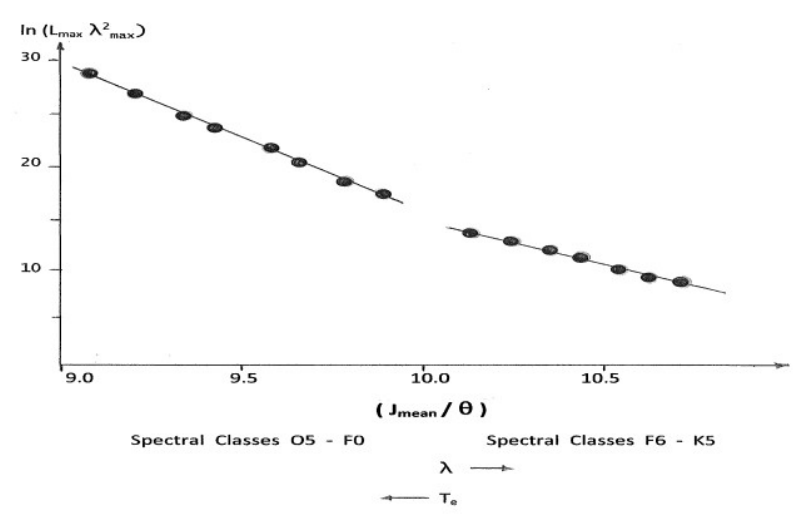

Fig $12 \ln \left(\mathrm{L}_{\max } \lambda_{\max }^{2}\right)$ plotted versus (1.6 $\left.\mathrm{J}_{\text {meanvalue }}\right) / \mathrm{hv} \mathrm{v}_{\max }$ for different stars from spectral classes $\mathbf{O}$ M ( Reproduction from Ref 33 )

\section{4b. The use of the Balmer lines}

In the paper Ref 32 it is shown in the that the intensity maximum of the continuous-and discrete spectra of stars seem to be the same, where the hydrogen Balmer absorption lines of different stars have been studied. These are the well known Planck curves with steep low wavelength side and a slow high wavelength side. The wavelength of the intensity maximum of continuous-and discrete spectrum seems to be the same. This in agreement with equation 1 and the new theory, where the Planck factor is a part of the new intensity formula. This is clearly seen in Fig 13 from the spectrum of two A-stars. The normalized flux is here proportional to the emissions from the continuous-and discrete spectrum. These curves show very good examples of Planck curves, where continuous-and discrete emissions seem to have the same wavelength maximum.

The wavelengths of the Balmer lines are shown in Table 3. By using equation 14 and Table 2 intensity ratios have been determined theoretically(from intensity formula) and experimentally by using the data of Ref 32 , from different spectral classes of stars. At the use of these intensity ratios $\mathrm{J}_{\mathrm{H}}=13.595 \mathrm{eV}$ for hydrogen was used. The electron tem- 
peratures for different spectral classes have earlier been determined in Tab 5 from Ref 33 . A summary of the values from the spectral classes of this paper is shown in Table 5. Nice correlation $(r=0.98)$ has been achieved between theoretical-and experimental intensity ratios.

This is shown in Fig 14 by the author and is, together with Fig 13 , a strong evidence of the fact that stars follow the new intensity formula, as atoms and ions do. Fig 14 shows very nice correlation between experiment and theory Refs 34, 35 and 36.

Table 3

Balmer lines used

$\mathrm{H}_{\alpha} \quad 6562.80 \AA$

$\mathrm{H}_{\beta} \quad 4861.32 \AA$

$\mathrm{H}_{\gamma} \quad 4340.46 \AA$

$\mathrm{H}_{\delta} \quad 4101.73 \AA$

$\mathrm{H}_{\varepsilon} \quad 3970.07 \AA$
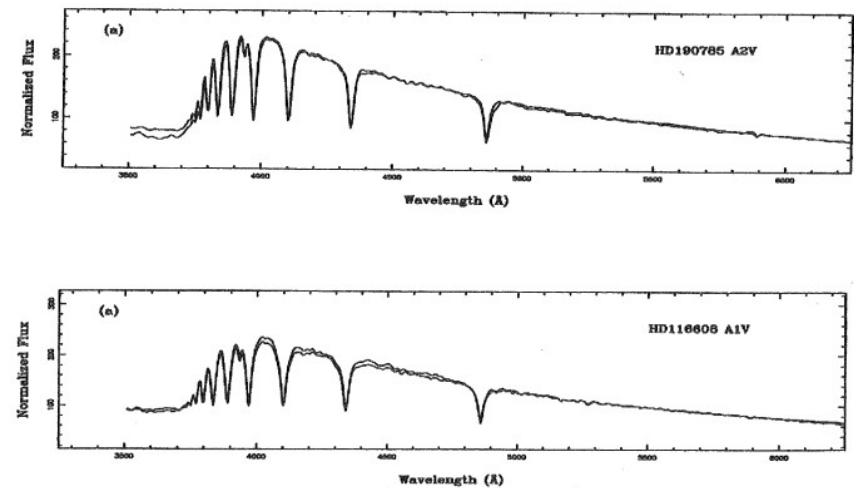

Fig 13 Plot of normalized flux versus the wavelength(Planck curve) for two different A-stars. The absorption hydrogen Balmer lines are clearly observed. The wavelength of the intensity maxima for both continuous and discrete emissions seems to be the same.

( Reproduction from Ref 34 )

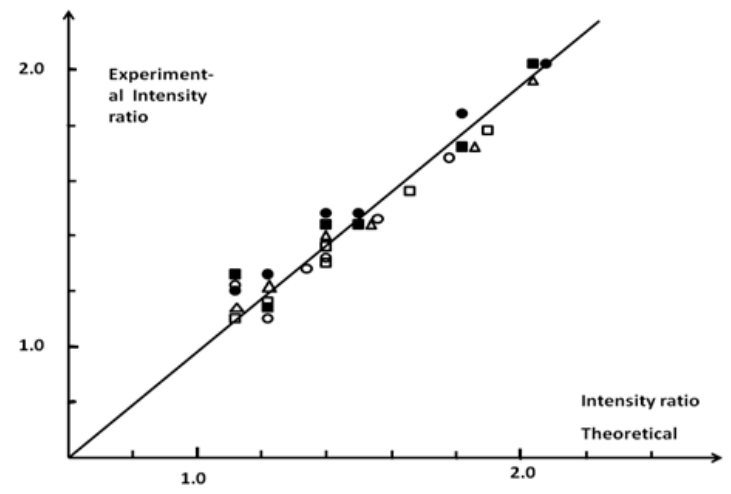

Fig 14 Spectral intensity ratios (experimental and theoretical) give very good correlation( $\mathrm{r}=0.98)$ using the Balmer lines from different spectral classes of stars using the new intensity formula. Spectral classes used : A8=unfilled circles , A5-A7=unfilled squares, A1-A3=unfilled triangles, B6V= filled circles, B3B4=filled squares. ( Reproduction from Ref 34 )

\section{4c. Determination of the effective temperature of a star}

In this section Table (66) p.564 in Ref 37 were then used, where the effective temperatures were tabled from many main sequence stars from different spectral classes (A-K). These effective temperature values were then plotted versus the electron temperature values from corresponding spectral class from Table 4 in this paper. This means that effective temperature values have been obtained for 12 main sequence stars and are tabled in Table 4.

Very nice correlation $(+-85 \mathrm{~K})$ is here obtained between the values from this investigation and the literature values based on the Stefan-Boltzmann temperature law and can be seen in Table 4 and Fig 15, which show good correlation ( $\mathrm{r}=0.99)$ Refs 34 and 36. 


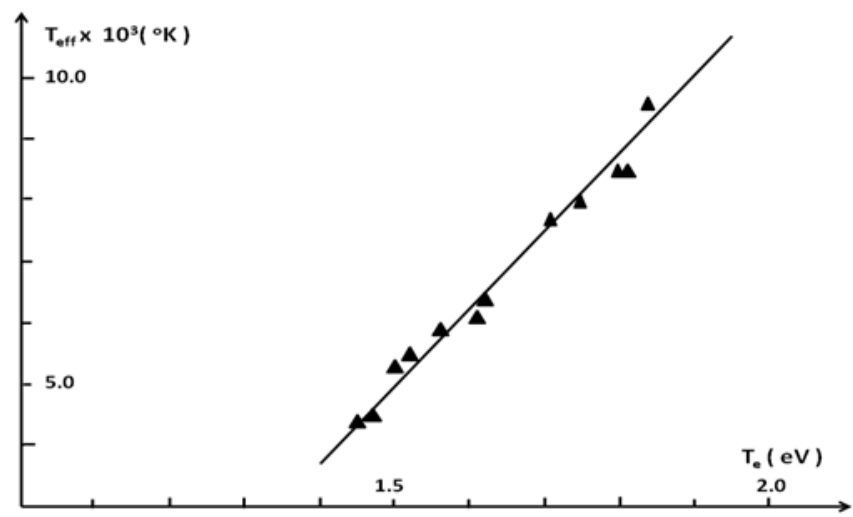

Fig 15 Effective temperature plotted versus electron temperature for a number of main sequence stars.(correlation $\mathbf{r}=\mathbf{0 . 9 9}$ ) (Reproduction from Ref 34 )

Table 4

\begin{tabular}{|c|c|c|c|}
\hline \multicolumn{4}{|c|}{ Determination of $T_{\text {effective of stars }}$} \\
\hline Frol & A the graph & From the literature & Spectral group \\
\hline Vega & $9300^{\circ} \mathrm{K}$ & $9300^{\circ} \mathrm{K}$ & $\mathrm{A} 0$ \\
\hline Altair & 8100 & 8000 & A7 \\
\hline Procoyon A & 7500 & 7500 & F5 \\
\hline Sun & 5700 & 5740 & G2 \\
\hline Sirius A & 9500 & 9700 & A1 \\
\hline Aldebaran & 4200 & 4100 & K5 \\
\hline Pollux & 4700 & 4500 & K0 \\
\hline Capella B & 5200 & 4940 & G5-G0 \\
\hline Regulus & 9700 & 10300 & B7 \\
\hline Canopus & 7250 & 7350 & F0 \\
\hline Fomalhaut & 8700 & 8500 & A3 \\
\hline Sirius B & 8400 & 8200 & A5 \\
\hline
\end{tabular}

\section{4d. Determination of the density of a star by using Balmer lines.}

The $\mathrm{C}$-factor of equation 1 is a product of factors of number densities of atoms and electrons. This means that by using the approximate formula of equation 1 we obtain :

$$
I=C \lambda^{-2} \exp \left(-(h v+J) / k T_{e}\right)
$$

Therefore we can express $\mathrm{C}$ as a function of the other parameters in equation 16 and by taking the ratio between the density of a star compared to the sun, we obtain the following expression

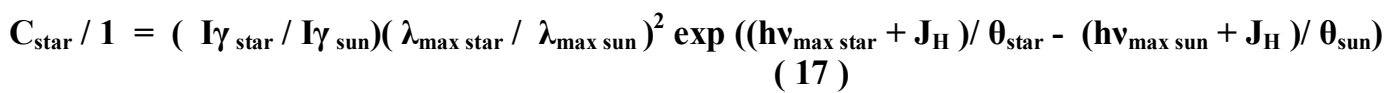

where $\mathrm{C}=1$ is the sun value and $\theta=k T_{e}$. The intensity ratio $\left(\mathrm{I} \gamma / \mathrm{I} \gamma_{\text {sun }}\right)$ here is the ratio between the $\gamma$-Balmer line from the star and the sun from the data of Ref (32). $\lambda_{\max }$ and the $h v_{\max }$ have also been taken from Ref (32) and the electron temperature values have been taken from Ref (33) for different spectral classes. $J_{\mathrm{H}}$ is the ionization energy of hydrogen.

The results of 12 stars here, are shown in Fig 16 and Table 5 where $\rho / \rho_{0}$-values been calculated for 12 different main sequence stars. In Fig 16 a straight line is achieved following in the near of the Schwarzschild line Ref 37 (p.555)( Refs 34 and 36 ).

\section{Table 5}

Determination of density ratio of 12 stars relative to the sun

$\begin{array}{lcc}\text { Star } & \rho / \rho_{\text {sun }}(\text { new method }) & \text { spectral clas } \\ \text { Aldebaran } & 1.26 & \text { K5 } \\ \text { Pollux } & 0.95 & \text { K0 } \\ \text { Capella B } & 0.91 & \text { G5 } \\ \text { Sun } & 1.0 & \text { G2 } \\ \text { Procyon A } & 0.66 & \text { F5 } \\ \text { Canopus } & 0.55 & \text { F0 }\end{array}$




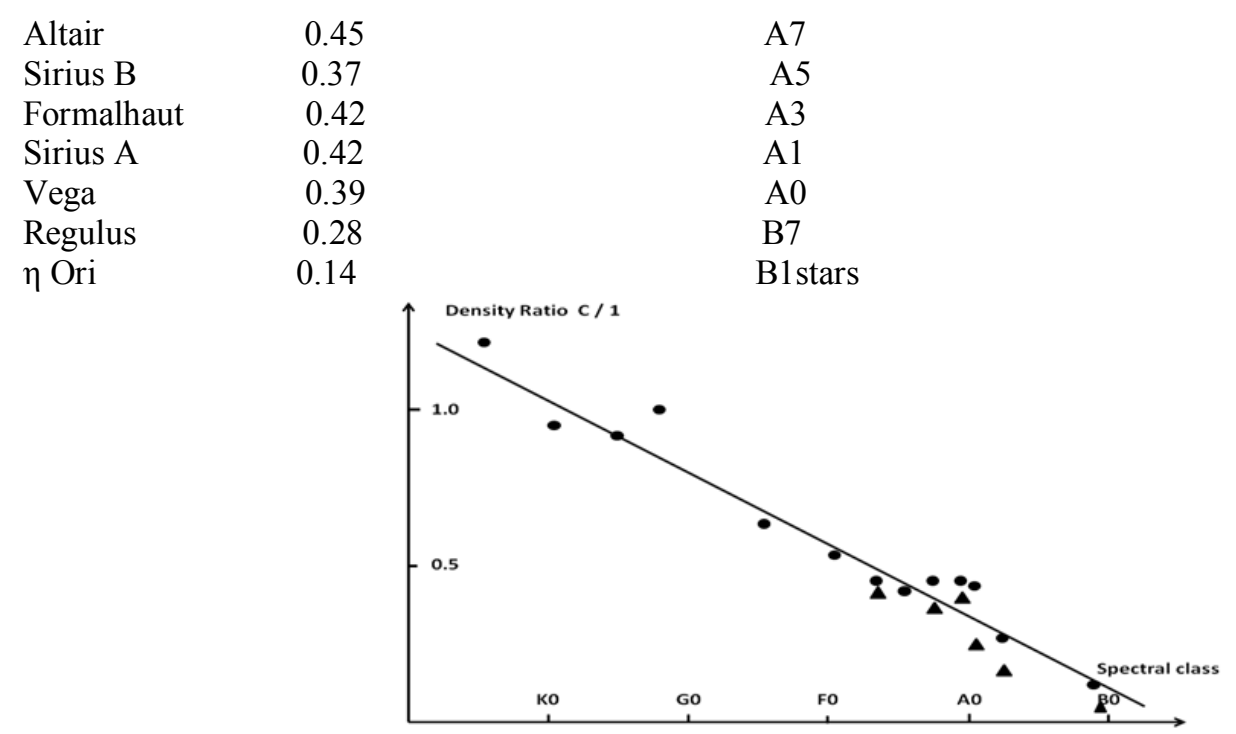

Fig 16 Density determination of stars relative to the sun at different spectral classes. Filled circles $=$ new method , Filled triangles $=$ Schwarzschild limit $($ Reproduction from Ref 34 )

\section{4e. Determination of the mass of the stars}

The usual Mass-Luminosity relation in astronomy means that there is a linear relationship between luminosity and mass of a star. Therefore there is a possibility to use the equation 1 in a similar way by the fact that $\mathrm{M} \alpha \mathrm{kT}_{\mathrm{e}}$. By using the approximate formula of equation 1 we obtain :

$$
I=C \lambda^{-2}\left(\operatorname{ex~} \mathbf{p}\left(-(\mathbf{J}+\mathbf{h v}) / \mathbf{k} \mathbf{T}_{\mathrm{e}}\right)\right.
$$

Therefore we can express $\mathrm{kT}_{\mathrm{e}}$ as a function of the other parameters in equation 18. By taking the ratio between the mass of a star compared to the sun $\left(\mathrm{M}_{0}\right)$, we obtain the following expression :

$\mathbf{M}_{\text {star }} / \mathbf{M}_{\mathbf{0} \text { sun }}=\ln \left(\mathbf{I} \lambda^{2} / \mathbf{C}_{0}\right)_{\text {max sun }}\left(J_{\text {mean star }}+\mathbf{h} v_{\text {max star }}\right) / \ln \left(\mathbf{I} \lambda^{2} / \mathbf{C}\right)_{\text {maxstar }}\left(J_{\text {mean sun }}+\mathbf{h} v_{\text {max sun }}\right)$

$$
\text { (19) }
$$

The $\ln \mathrm{I} \lambda^{2}, \mathrm{C}, \mathrm{J}_{\mathrm{med}}$ and $\mathrm{h} v_{\max }$ - values in equation 19 can be determined from Fig 12 and Table 2 in this paper. The $\ln \left(\mathrm{I} \lambda^{2}\right)$-values can be shown directly from the graph in Fig 12 for a certain star and the $\mathrm{C}$-values are shown as the prolongation of the two lines in Fig 12 for a certain star placed on one of the lines. The $\mathrm{M} / \mathrm{M}_{0}-$ values have been tabled in Table 6 for 10 different stars, which show good agreement with the literature values. This very nice agreement is also shown in Fig 17 between $\left(\mathrm{M} / \mathrm{M}_{0}\right)$ - values from this new method and literature values and show a nice linear relationship ( $\mathrm{r}=0.97$ ) Ref 34 .

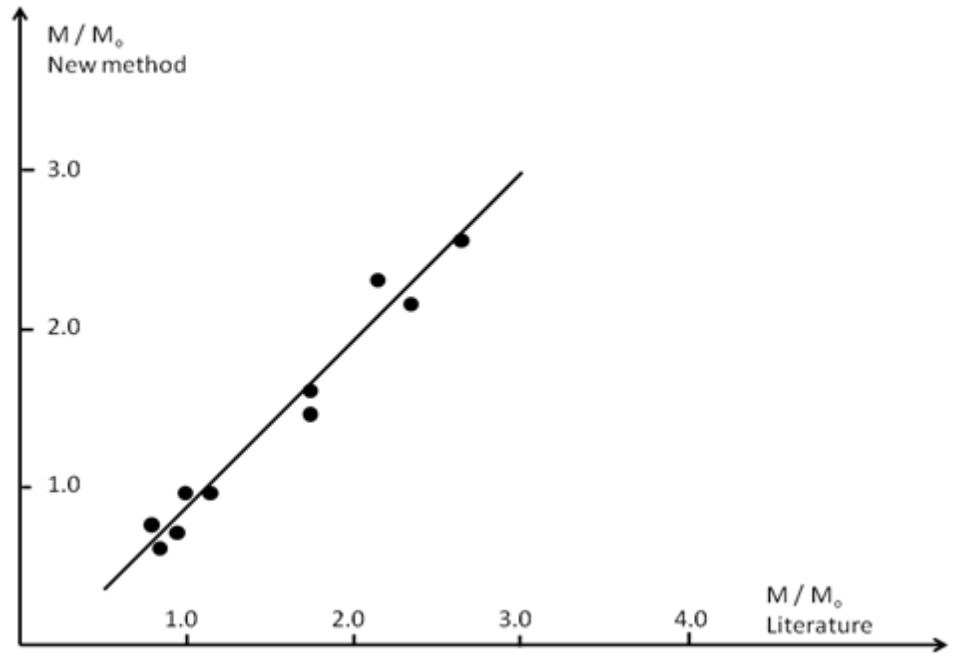

Fig 17. Determination of the mass of a number of stars with the new method together with literature values. Correlation r= 0.97 . ( Reproduction from Ref 34 ) 


\section{Table 6}

$\begin{array}{lccr}\text { Determination of the mass ratio relative to the sun } & \\ \text { Star } & \mathbf{M} / \mathbf{M}_{\mathbf{o}} \text { (new method) } & \mathbf{M} / \mathbf{M}_{\mathbf{o} \text { (Literature) }} & \text { Spectral class } \\ \text { Vega } & 2.55 & 2.50 & \mathrm{~A} 0 \\ \text { Formalhaut } & 2.15 & 2.30 & \mathrm{~A} 3 \\ \text { Sirius A } & 2.35 & 2.10 & \mathrm{~A} 1 \\ \text { Altair } & 1.62 & 1.70 & \mathrm{~A} 7 \\ \text { Dubhe } & 1.40 & 1.70 & \mathrm{~F} 0 \\ \alpha \text { Centauri A } & 1.00 & 1.10 & \mathrm{G} 2 \\ \text { Sun } & 1.00 & 1.00 & \mathrm{G} 2 \\ \text { Capella B } & 0.76 & 0.80 & \end{array}$

\section{Discussion}

This paper shows that the new intensity formula seems to be applicable to many different kinds of optical light sources at different experimental conditions and temperatures from all the methods shown in this paper. This paper is an extended version of two earlier review papers concerning this new intensity formula. The fluctuation analysis of spectral line intensity ratios in optical emission spectroscopy is a very sophisticated method of sorting the correct formula, where questions concerning photon efficiency versus wavelength for spectrometer systems, can be eliminated.

The absolute intensity method show linearity over $25 \mathrm{eV}$ for $\mathrm{C}(11)$ - lines and $14 \mathrm{eV}$ for C(I)-lines. This is impossible to achieve without a correct intensity formula. Deviations caused by photon efficiency versus wavelength for spectrometer systems are here very small in these graphs. The most dominant linearity factor is a correct exponent in the intensity formula.

In this paper the absolute intensity method shows good linearity for 17 atomic elements and 11 ionic elements in the energy ranges $15 \mathrm{eV}$ for atoms and up to $50 \mathrm{eV}$ for ions and up to $200 \mathrm{eV}$ for higher ions. This is impossible to achieve without a correct intensity formula. The most dominating linearity factor is a correct exponent in the intensity formula. Deviations caused by photon efficiency versus wavelength for spectrometer systems are here very small in these graphs.

The electron temperatures ( $\theta$-values) determined in this paper are in accordance with second electron measurements from the literature. There it is possible to study the mean values of half widths of the energy distributions $f\left(E_{e}\right)$ of secondary electrons emitted from different metal surfaces under impact of inert gas ions. This half width is increased with ions from higher ionization states.

Both these measurements gave temperatures around $2 \mathrm{eV}$ for atoms (17 elements) and around $4 \mathrm{eV}$ for ions(11 elements) and are in accordance to secondary electron measurements, which are a strong support of the intensity formula. The nice linearity using the absolute intensity method for higher ions of oxygen and neon is a strong evidence of the general recursion formula in equation 8 , which includes the $\Sigma \mathrm{J}$ - term, and has the same structure as the new intensity formula in equation 1.

The results in Ref 26 about the photoelectric effect explain the general appearance of the shape of the current versus frequency expression. By using the inverse of the approximate intensity formula in equation 1, a solution about relationship between current and frequency has been achieved. This inverse is shown in equation 11 and is demonstrated in Fig 8, where a nice relationship between literature experiment and the new theory were achieved.

Fig 9 is a strong evidence of the J-term of equation 11. From this graph it is possible to study the $\mathrm{J}$ dependence of 22 different elements(metals). The "knee" of this curve depends of the heating effect at higher frequencies. By expressing $\mathrm{J}$ as a function of $\lambda$ as in equation 13 will also lead to a strong support of equation 11.

The $\mathbf{J}$-term in equation 11 is also supported by different breakdown experiments with lasers. In these experiments the electrons were created by the photoeffect from strong laser beams. The breakdown pressure is here proportional to the number density of electrons(current) and therefore it will follow equation 11.

Alkali metals were also studied in a similar graph in Fig 10. The heating effect here is not so high and the general appearance is the same as the other graphs.

The internal electron temperature was also determined in relation to the ionization energies. Intensity studies were also done in the ICP-plasma, where the internal electron temperature was studied at different heights in the plasma supporting the new intensity formula.

Studies from the LED-and flourescens experiments at room temperature were also discussed

in relation to the Planck profile of the new formula.

Breakdown- and X-ray experiments have also been discussed in relation to equations 11 and 1 . These formulas constitute new formulas for breakdown experiments and X-ray emissions. 
In the nature the new formula and the fluctuation method have been tested in Ba-release experiments in the upper atmosphere using rockets and spectrophotometers on the ground. Airglow and auroral emissions were also measured on ground measurements with spectrophotometer, where the optical emission mechanism was studied. These results were in agreement to the new intensity formula.

The absolute intensity method of analysis has shown to be a simple method of verifying the new intensity formula by using atomic, ionic and stellar data. By using this method together with the new intensity formula it has been possible to determine the mean electron temperature in different laboratory plasmas and in the optical layers of a star without knowing so much about the chemical composition of the star. These mean electron temperature values fit well with other methods from the literature. The method also gives an organizing method for stars similar to the established HR-diagram. The $\mathrm{J}_{\text {meanvalue }}$ has shown to be a kind of "signum" for every star. Fig 12 has shown to be a valuable and simple method of organizing and classifying the stars without knowing so many other details about the stars. The Balmer spectral absorption lines seem to follow the new intensity formula too, which is clearly seen in Figs 13 and 14. This is clearly seen by the correlation coefficient ( 0.98). This means that discrete emissions in the star do follow the new intensity formula but are heavily absorbed in the star. Therefore, the light coming from the star is mostly continuous radiation following Planck radiation law.

It has also been possible to determine the effective temperature of a number of stars from different spectral classes on the main sequence. The results gave good agreement with the established temperature method by Stefan-Boltzmann.

The density of a number of stars was also determined compared to the sun, from different spectral classes on the main sequence. These values are in accordance with the Schwarzschild limit. The graph in Fig 16 shows a nice linear relationship.

It was also possible to determine the mass of a number of stars compared to the sun from different spectral classes on the main sequence. These values are in accordance with the literature values. The graph in Fig 17 shows a nice linear relationship.

\section{Acknowledgement :}

I express a big gratitude to my friend and collegue Dr. Sten Yngström at the Swedish Institute of Space Physics for inspiring and skillful cooperation during many years. I also gratitude Prof. Bengt Hultqvist at Swedish Institute of Space Physics for his encouraging support of this work in the beginning of this project.

[1]. Yngström S., Int. J. Theo. Phys. 33 (7): 1479, (1994)

[2]. Thelin B., Eurasian Journal of Analytical Chemistry, Vol.4, Nr 3, s.226-233, (2009)

[3]. Thelin B., African Phys Review, 4 (2010), 121

[4]. Thelin B., Indian Journal of Pure \& Applied Physics, Vol 50, (April 2012),231

[5]. Thelin B.,ICP Information. Newsletter, 10, 835 (1985)

[6]. Thelin B.,Analyst 111, 419, (1986)

[7]. Thelin B.,Analyst 112, 623, (1987)

[8]. Thelin B., Talanta 4, 317 (1988)

[9]. Thelin B., Can. J. Spectrosc. 33, 94 (1988)

[10]. Yngström S and Thelin B., Appl. Spectrosc. 44, No 9, 1566 (1990)

[11]. Thelin B. and Yngström S., Spectrochim. Acta 41B, 403 (1986)

[12]. Thelin B , Appl. Spectrosc. 44: 818. (1990)

[13]. W.F. Meggers, C.H. Corliss, and F. Scribner, Tables of Spectral Line Intensities, National Bureau of Standards ,Monograph 32 Part 1,Washington D.C. (1961)

[14]. Striganov A. R. and Sventitski N.E., Tables of Spectral Lines of Neutral and Ionized Atoms., IFI/Plenum, New York, (1968)

[15]. M.Kaminsky, Atomic \& Ionic Impact Phenomena on Metal Surfaces, Springer Verlag, Berlin, Heidelberg, New York, 1965

[16]. Yngström S, Appl. Spectrosc. 48 (5): 587. (1994)

[17]. Thelin B., African Review of Physics, 7, 31 (2012)

[18]. Thelin B., African Review of Physics, 7, 459 (2012)

[19]. Blades M W, Horlick G, Spectrochim. Acta 36B (9), 861. (1981)

[20]. Li F., Son D.I., Kim T.W., Dong W. and Kim Y.H., Jpn J. Appl. Phys. 47 (6), 5086 (2008)

[21]. Choi J., Jhin J. Yang S. Baek J. Lee J. and Byun D., Jpn. J. Appl. Phys. 47 (11), 8265 (2008)

[22]. Ohshima Y, Kohn H, Lim E, Manaka T, Iwamoto M , Jpn. J. Appl. Phys. 47(2):1297. (2008),

[23]. Meek J.M. and Cragg J.D., Electrical Breakdown of Gases, New York, Brisbane, Toronto (1978)

[24]. Comton A.H. and Allison S.K., X-ray in Theory and Experiment, van Nostrand Company Inc., Princeton, New Jersey, Toronto, London (1935)

[25]. Flugge S., Handbuch der Physik, Springer Verlag, Berlin, Göttingen, Heidelberg, (1957)

[26]. Thelin B., IOSR Journal of Applied Physics (IOSR-JAP) Vol 3 (5) p.59 (2013)

[27]. Hughes, A. L. and Du Bridge L.A., Photoelectric Phenomena, (1932)

[28]. Thelin B.,In Proceedings of the $16^{\text {th }}$ Annual Meeting on Atmospheric Studies by Optical Methods, Oulu, Finland, Tanskanen P. ed. (1988)

[29]. Dick K.A., Barium releases at altitudes between 200 and 1000 km, A joint Max- Planck Institute-Nasa experiment, p.47,National Aeronautic and Space Administration, Washington D.C.(1971) 
[30]. Thelin B., In Proceedings of the $14^{\text {th }}$ Annual Meeting on Atmospheric Studies by Optical Methods ,M.J.Rycroft, ed, Cambridge, England (1986)

[31]. Barbier D., The airglow and the aurora, Pergamon Press London and New York, p.38(1955)(Symposium in Belfast September 1955)

[32]. Silva D.R. and Cornell M.E., Astrophysical Journal Suppliment Series, 81, No2, Aug. (1992)

[33]. Thelin B., Fizika B, 19, 4 (2010) 329.

[34]. Thelin B., IOSR Journal of Applied Physics(IOSR-JAP) 3, Issue 6 (2013) 42

[35]. Thelin B., Indian Journal of Pure \& Applied Physics 51, (March 2013) p.174

[36]. Thelin B., IOSR Journal of Applied Physics(IOSR-JAP) 2, Issue 5 (2012) 48

[37]. Lang K.R., Astrophysical Formula, Springer Verlag, Berlin, Heidelberg, New York, (1974) 